\title{
REALIDADE VIRTUAL NO TREINAMENTO DO EQUILÍBRIO EM IDOSOS \\ - um estudo de revisão
}

\author{
Eujessika Katielly Rodrigues Silva ${ }^{a}$ \\ Lorena Carneiro de Macêdo ${ }^{b}$
}

\begin{abstract}
Resumo
O processo natural de envelhecimento desencadeia no indivíduo uma série de alterações fisiológicas. As manifestações dos distúrbios do equilíbrio corporal é uma das principais alterações desse processo e pode levá-los à redução de sua autonomia social, pela predisposição a quedas. O objetivo do presente estudo foi verificar a atuação da realidade virtual no treinamento do equilíbrio em idosos mediante a utilização de jogos virtuais como forma de tratamento para esses pacientes. Trata-se de uma revisão de literatura através da consulta aos bancos de dados PubMed/MEDLINE; Lilacs/SciELO; Cochrane, utilizando como descritores, correspondentes em português e inglês, os seguintes termos: "balance" and "virtual reality" and "older" or "elderly" or "aging". Foram encontrados na literatura pesquisada poucos estudos abordando essa temática, porém, os estudos intervencionistas realizados com a população idosa oferecem resultados favoráveis à melhoria do equilíbrio de pacientes idosos mediante o treinamento com realidade virtual. Entretanto, ainda existe uma lacuna na literatura relacionada à qualidade das pesquisas publicadas, já que são poucos os ensaios clínicos randomizados e as revisões sistemáticas de literatura, havendo a necessidade de mais pesquisas na área com a finalidade de aumentar o rigor científico e contribuir para a disseminação do conhecimento nessa temática.
\end{abstract}

Palavras-chave: Equilíbrio Postural; Envelhecimento; Terapia de exposição à realidade virtual.

a. Membro do Núcleo de Estudos da Motricidade Humana (MOTRIS); Monitora de Fisioterapia Desportiva; Graduanda em Fisioterapia pela Universidade Estadual da Paraíba (UEPB). eujessika_my@hotmail.com

b. Mestre em Saúde Materno Infantil pelo Instituto de Medicina Integral Prof. Fernando Figueira (IMIP); Pós-graduada em Fisioterapia Manual pela Faculdade Maurício de Nassau (FMN); Quiropraxista (IBRATES); Membro do Núcleo de Estudos da Motricidade Humana (MOTRIS); Graduada em Fisioterapia pela UEPB. lorenacmacedo@gmail.com 


\title{
VIRTUAL REALITY IN BALANCE TRAINING IN THE ELDERLY
}

- a revision study

\begin{abstract}
The natural aging process triggers a series of physiological changes in the individual. The onset of body balance disorders is one of the main changes in that process and may cause the person's loss of independence, due to the predisposition to falls. This study aims to verify the role of virtual reality for balance training in the elderly with electronic games as a form of treatment for these patients. This is a literature review through the query of the PubMed/MEDLINE; Lilacs/SciELO; and Cochrane databases, using the following terms, with their corresponding Portuguese translation: "balance" and "virtual reality" and "older" or "elderly" or "aging." A few studies were found in the revised literature that address this theme, but the interventionist studies carried with the elderly offer favorable results to the improvement of balance in the elderly through the training with virtual reality. However, there is still a gap in the literature concerning the quality of published researches, since there are few randomized clinical essays and systematic literature reviews and thus, there is a need for more researches in the area aiming to increase the scientific rigor and contribute to the dissemination of knowledge on this theme.
\end{abstract}

Keywords: Postural balance, Aging, Virtual reality exposure therapy.

\section{INTRODUÇÃO}

O sistema de controle postural, que integra as funções do sistema nervoso, sensorial e motor, é responsável pela manutenção do equilíbrio do corpo. O sistema sensorial fornece informações sobre a posição de segmentos corporais em relação a outros segmentos e ao ambiente. Essas informações sensoriais são levadas ao sistema nervoso central (SNC) e são integradas ao sistema motor, gerando respostas neuromusculares, para produzir os movimentos corporais. O resultado dessa integração entre o sistema sensorial e o sistema motor produz um maior ou menor grau de controle do equilíbrio.(1)

Essas respostas neuromusculares são importantes para garantir que o centro de gravidade do corpo seja mantido dentro da base de sustentação sem que as oscilações e variações do centro de gravidade provoquem grandes instabilidades, permitindo ao indivíduo manter-se em equilíbrio em situações estáticas e durante a realização de movimentos.(1)

Porém, com o progredir da idade, o indivíduo pode vir a sofrer com o comprometimento dos sistemas citados anteriormente, e várias etapas do controle postural podem ser extintas resultando em uma diminuição na habilidade do idoso em retornar ao seu estado inicial após alguma perturbação. No idoso, as estratégias utilizadas pelo corpo para responder as variações e oscilações do centro de gravidade encontram-se afetadas, podendo acarretar o desequilíbrio. ${ }^{(2)}$

O déficit do equilíbrio é um dos principais fatores limitantes na vida dos idosos. Em mais da metade dos casos a diminuição do equilíbrio tem origem entre 65 e 75 anos, em 80\% dos casos não há uma causa especifica. Esse déficit pode levar os idosos à 
redução de sua autonomia social, pela predisposição a quedas e risco de fraturas, trazendo sofrimento, imobilidade, medo e altos custos com o tratamento de saúde. ${ }^{(3)}$

Por ser um evento frequente em pessoas idosas, a queda constitui um grave problema de saúde pública, sendo o tipo de acidente mais frequente no idoso. Suas complicações estão associadas a principal causa de morte nos maiores de 65 anos. Por isso há a necessidade de políticas públicas direcionadas a essa população e de estudos voltados para prevenção, tentando minimizar as quedas e suas consequências. ${ }^{(2)}$ Para minimizar a ocorrência das é importante que seja realizado um treinamento do equilíbrio direcionado às limitações físicas dos idosos. ${ }^{(4)}$

As modalidades de intervenção terapêutica para o treino do equilíbrio não devem ser utilizadas de forma isolada e devem ser baseadas na avaliação criteriosa das dificuldades e comprometimento de cada paciente. $O$ treinamento do equilíbrio deve envolver vários sistemas (sensorial, musculoesquelético, cognitivo-perceptual, emocional, neuromuscular e outros) que trabalham de forma cooperativa para controlar a orientação e estabilidade do corpo. ${ }^{(5)}$

Para condições ideais do treino do equilíbrio, o idoso deve está com o sistema emocional estabilizado, já que condições como o pânico, o medo, a ansiedade e a depressão têm um impacto negativo sobre o equilíbrio corporal. Além disso, é importante uma boa cognição do paciente, já que para a execução dos exercícios de treinamento é necessário haver concentração, para que os movimentos sejam executados de forma isolada e associados a tarefas motoras específicas. ${ }^{(5)}$

Embora os exercícios terapêuticos convencionais utilizados para o treinamento do equilíbrio, sejam variados e simulem atividades da vida diária, muitos dos idosos se queixam da monotonia do tratamento, já que não conseguem perceber um resultado imediato da proposta terapêutica tradicional, não tendo parâmetros para compreender sua evolução mediante os exercícios realizados. ${ }^{(6)}$
Dessa maneira, a realidade virtual surge como uma tecnologia inovadora que está sendo utilizada para o tratamento de diferentes tipos de pacientes. (7) A correção do equilíbrio é um dos benefícios associado a esse contexto terapêutico, já que através de jogos virtuais o paciente pode interagir com o ambiente virtual proposto, recebendo um feedback visual em relação às mudanças de seu movimento, e deste modo criar estratégias para recuperar e/ou manter o equilíbrio. ${ }^{(8)}$

A aplicação da realidade virtual aumentada (RVA) atua na melhoria do controle postural e na minimização de quedas em pessoas idosas, o que demonstra efetividade e potencialidade das aplicações dos serious games nessa parcela da população ${ }^{9}$

Serious Games é uma classe de jogos com um propósito específico, voltados ao aprendizado e ao treinamento. $\mathrm{Na}$ área da saúde, os serious games podem ser classificados nas seguintes categorias: 1) auxiliares de terapia; 2) promoção da saúde e condicionamento físico; 3) monitoramento da saúde; e 4) treinamento. Para fins de treinamento, os serious games são aplicados para simular situações críticas, que envolvam algum tipo de risco, tomada de decisões, ou para desenvolver habilidades específicas. Esses jogos ultrapassam os conceitos do entretenimento e proporcionam uma aproximação dos avanços da realidade virtual aumentada com a sociedade, visam principalmente a simulação de situações práticas, com abordagem atraente e lúdica, favorecendo a absorção de habilidades psicomotoras $^{(10)}$.

Nas ciências da saúde, cresce cada vez mais a realização de estudos que possam trazer benefícios à população idosa, para que novas abordagens de reabilitação possam minimizar as alterações negativas que acontecem no envelhecimento, melhorando a qualidade de vida dos idosos. Desse ponto de vista, o treinamento do equilíbrio com a Realidade Virtual (RV) se apresenta como uma inovação para o tratamento e pode trazer benefícios mediante adesão prazerosa dos idosos, diminuindo o risco de quedas nessa parcela da população, podendo contribuir para atenuar esse problema de saúde pública. ${ }^{(1)}$ 
Dessa forma, o objetivo do presente estudo foi verificar, através de revisão na literatura, a ação da realidade virtual no treinamento do equilíbrio em idosos mediante a utilização da realidade virtual como forma de tratamento.

\section{MÉTODOS}

Trata-se de uma revisão de literatura com consulta aos bancos de dados PubMed/MEDLINE (19662013); Lilacs/SciELO (1982-2013); Cochrane (19932013). Sendo utilizados como descritores correspondentes em português e inglês, os seguintes termos: "balance" and "virtual reality" and "older" or "elderly" or "aging".

A pesquisa foi realizada entre setembro de 2013 a janeiro de 2014, nas línguas portuguesa e inglesa, sendo incluídos todos os estudos realizados com equilíbrio em idosos e que em sua reabilitação durante algum momento fosse utilizada a realidade virtual, independente do ano de sua publicação. Foram considerados inadequados para esta pesquisa, estudos que abordassem o treinamento com realidade virtual em patologias associadas ao envelhecimento, e estudos com o treinamento do equilíbrio em idosos sem o uso da realidade virtual.

\section{RESULTADOS E DISCUSSÃO}

$\mathrm{Na}$ presente revisão, foram analisados seis artigos completos que atenderam aos critérios de inclusão e exclusão pré-determinados. Os resultados de cada artigo foram sumarizados e expostos nos parágrafos seguintes.

Em um estudo realizado em 2012, mulheres na pós-menopausa foram submetidas a um programa de treinamento do equilíbrio com o Nintendo Wii

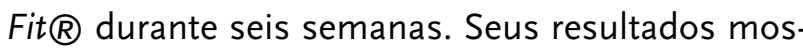
traram que houve uma melhoria do equilíbrio com resultados satisfatórios na mobilidade, agilidade e funcionalidade, havendo diminuição do risco e do medo de quedas na amostra estudada. ${ }^{(6)}$
Um estudo realizado com seis idosos utilizou jogos que exploravam movimentos laterais e ântero-posteriores, com o uso do console Nintendo Wii Balance Board@. A avaliação foi feita através da Escala de Tinetti e através da própria plataforma do console. Foram realizados dez atendimentos com os jogos, tendo duração de 20 minutos cada atendimento, no período de quatro semanas. Os autores encontraram uma melhora do equilíbrio que refletiu em uma maior confiança na capacidade dos idosos em realizar tarefas funcionais. Terminados os dez atendimentos, todos os participantes relataram ter gostado da experiência e demonstraram interesse em continuar realizando treinamento com a Realidade Virtual. ${ }^{(12)}$

Outra pesquisa avaliou as alterações do equilíbrio em 40 idosos, sendo a maioria do sexo feminino, mediante uso do Nintendo Wii Balance Board $(\mathbb{B}$. Os participantes foram divididos em dois grupos, um grupo recebeu intervenção com uso da realidade virtual, através do Nintendo Wiiß, e o outro grupo não recebeu nenhum tipo de intervenção. Durante seis semanas o grupo experimental foi submetido a um programa de treinamento utilizando jogos virtuais, cada sessão durava 35-45 minutos, sendo realizadas três vezes por semana, totalizando 18 sessões. E foi encontrada melhora no equilíbrio dinâmico e estabilidade postural, aumento na confiança para a realização de atividades funcionais e redução dos riscos de queda. É importante destacar que nenhum dos participantes foi capaz de completar toda a série de exercícios com independência total, sendo necessário algum tipo de apoio (como cadeira, andador, ou mesmo a mão do fisioterapeuta) para maior segurança. ${ }^{(7)}$

Em um ensaio clínico realizado com 32 idosos, divididos em dois grupos, avaliou-se o equilíbrio e a força muscular do quadril. O grupo controle não sofreu nenhum tipo de intervenção. $O$ grupo experimental foi submetido a um programa de treinamento, com o uso da realidade virtual por meio do Kinect $X$ box $360 ®$, sendo realizados 24 atendimentos, com duração de uma hora e frequência de três vezes por semana. A força muscular do quadril foi 
medida usando dinamômetro multimodal, observando os movimentos de flexão, extensão, abdução e adução do quadril. Os participantes foram orientados no primeiro treino e em seguida desenvolveram os exercícios sem supervisão durante oito semanas. Os movimentos prioritários no programa de exercício eram os movimentos de membros inferiores. Após as oito semanas de prática de exercícios com os jogos virtuais foi observada uma melhora significativa na força muscular e também no equilíbrio dos indivíduos submetidos ao uso da realidade virtual como forma de tratamento. ${ }^{(13)}$

Outro estudo desenvolvido com 32 idosos buscou avaliar equilíbrio, mobilidade, flexibilidade e quedas utilizando o videogame associado a Balance Board. A amostra foi dividida em dois grupos que foram submetidos a dez atendimentos, duas vezes na semana, com duração de 30 minutos. O primeiro grupo recebeu a intervenção pelo treinamento proprioceptivo convencional e o segundo grupo pelo treinamento proprioceptivo com realidade virtual. Como resultado do estudo, observou-se que o treinamento proprioceptivo com realidade virtual mostrou ser mais eficiente que o treinamento proprioceptivo convencional em indivíduos idosos pelas variáveis de POMA (Performance Oriented Mobility Assessment) e Escala Unipodal.(14)

No estudo realizado com 10 indivíduos para a avaliação da funcionalidade, equilíbrio (através da escala de Berg) e qualidade de vida em idosas utilizando jogos do console Nintendo Wiiß, o protocolo de treinamento consistiu em oito sessões, com 60 minutos de duração, realizados duas vezes por semana. Os resultados mostraram que o treinamento com o Nintendo Wiiß não promoveu melhora significativa do escore obtido pela avaliação do equilíbrio a partir da escala de Berg, porém manteve o nível elevado do escore total das voluntárias. Os autores destacam a associação positiva do Nintendo Wi® com o tratamento, já que houve a manutenção desses valores após a intervenção".

Como visto no decorrer do presente artigo, o treinamento do equilíbrio através da realidade virtual é feito adotando-se exercícios semelhantes aos realizados no treinamento convencional, a diferença está na interatividade que há entre o paciente e o jogo virtual. Essa interatividade oferece uma abordagem atraente e lúdica para a prática dos exercícios de equilíbrio e funciona como incentivo já que a atividade se torna desafiadora e prazerosa. $^{(8,10)}$

Essa forma de tratamento foge aos métodos convencionais de reabilitação, oferece ao jogador (idoso) uma interação com a realidade virtual, através do feedback somatossensorial e do feedback visual imediato, fazendo com que estratégias sejam criadas para a recuperação/manutenção do equilíbrio. Além disso, cada partida fornece ao jogador uma resposta imediata do seu desempenho no jogo através da pontuação indicada ao fim de cada partida, o que pode funcionar como estímulo para que o paciente deseje superar os seus limites buscando uma pontuação cada vez maior. . $^{(8,11,15)}$

O uso da realidade virtual oferece estímulo aos sistemas sensoriais, no momento em que o paciente (idoso) deve olhar os movimentos simulados virtualmente na tela de projeção, esses estímulos sensoriais são integrados no SNC e convertidos em respostas motoras, no momento em que o paciente reproduz os movimentos virtuais na sua realidade corporal. Dessa forma, percebe-se que o tratamento com realidade virtual atua no sistema motor postural contribuindo para o treinamento, recuperação e manutenção do equilíbrio.

Ficou comprovado que o uso dos jogos virtuais como forma de treinamento do equilíbrio em idosos, promoveu melhora ou manteve o nível de equilíbrio na população estudada, bem como, estabeleceu nesses indivíduos, o aumento da confiança para a realização de suas atividades de vida diária, mobilidade, funcionalidade e outros fatores que são imprescindíveis a essa população.

Os resultados encontrados mostram que os jogos virtuais estão atrelados com o componente motivacional que estimula a sua prática e a torna mais prazerosa, sendo um grande aliado na reabilitação cinético-funcional dos indivíduos. Mesmo assim, apesar da dimensão que a temática sobre 
realidade virtual está atingindo, ainda há a necessidade de outras investigações que possam comprovar e assegurar ainda mais, os benefícios do treinamento com realidade virtual no equilíbrio dos idosos.

Percebe-se que o treinamento com a Realidade Virtual oferece resultados favoráveis à meIhoria do equilíbrio de pacientes idosos. Mesmo assim, ainda existe uma lacuna na literatura relacionada à qualidade das pesquisas publicadas, já que são poucos os ensaios clínicos randomizados e as revisões sistemáticas da literatura (estudos com nível de evidência I).

Os estudos encontrados demostram que o uso da realidade virtual pode ser considerado um valioso instrumento para o tratamento fisioterapêutico de pacientes com disfunções motoras relacionadas à diminuição do equilíbrio, oferecendo um diferencial na terapêutica proposta pela fisioterapia convencional, já que há uma maior motivação e adesão do paciente a esse tipo de tratamento.

Em relação à acessibilidade da Realidade Virtual para o serviço fisioterapêutico, vale ressaltar que existem aparelhos de videogame disponíveis no mercado, com fácil manuseio, apresentando baixo custo quando comparado a outros recursos e que podem ser inseridos facilmente no ambiente clínico por ser um instrumento compacto e portátil.

No treinamento que visa melhorar o equilíbrio, o idoso é desafiado a realizar movimentos que oferecem episódios de instabilidade para que com isso eles possam aprender estratégias necessárias para manter o equilíbrio corporal. Diante disso, ressaltamos a importância da conscientização de que qualquer tipo de treinamento com a Realidade Virtual deve ser supervisionado por um fisioterapeuta. Além dos objetivos do tratamento, que são traçados pelo profissional da fisioterapia, os movimentos realizados devem ser executados de maneira adequada para que não haja o risco de ocorrer lesões em outras estruturas corporais, sendo imprescindível que os pacientes respondam aos comandos desse profissional.
Apesar de os resultados existentes nos estudos atuais se apresentarem favoráveis, o número de trabalhos acerca dessa abordagem ainda é reduzido. A realização de pesquisas futuras pode encontrar resultados importantes para aperfeiçoar o rigor cientifico da área, aumentando a qualidade das informações e atribuindo evidências ao campo da recuperação do equilíbrio em idosos através da Realidade Virtual.

\section{REFERÊNCIAS}

1. Duarte M, Freitas SMSF. Revisão sobre posturografia baseada em plataforma de força para avaliação do equilíbrio. Rev Bras Fisioter. 2010;14 (3):183-92.

2. Maciel AC, Guerra RO. Prevalência e fatores associados ao déficit de equilíbrio em idosos. R. bras. Ci e Mov. 2005;13 (1):37-44.

3. Ruwer SL, Angela GR, Larissa FS. Equilíbrio no Idoso. Rev Bras Otorrinolaringol. 2005;71(3):298-303.

4. Rebelatto JR, Morelli JGS. Fisioterapia geriátrica. 2. ed. Barueri: Manole; 2007.

5. Perracini MR, Fló CM. Funcionalidade e envelhecimento. Rio de Janeiro: Editora Guanabara Koogan, Série Fisioterapia: Teoria e Prática Clínica. 2009.

6. Singh DKA, Rajaratnam BS, Palaniswamy V, Pearson H, Ramana VP, Bong OS. Participating in a virtual reality balance exercise program can reduce risk and fear of falls. Journal Elsevier. 2012;73 (3):1-5.

7. Rendon AA, Lohman EB, Thorpe D, Johnson EG, Medina E, Bradley B. The effect of virtual reality gaming on dynamics balance in older adults. Age Agening. 2012; 41: 549-552.

8. Schiavinato AM, Cristiano B, Lilian M, Liliane SL. Influência do Wii Fit no equilíbrio de paciente com disfunção cerebelar: estudo de caso. J Health Sci Inst. 2010;28 (1):50-52.

9. Virk S, McConville KMV. Virtual Reality Applications in Improving Postural Control and Minimizing Falls. ConfProc IEEE Eng Med Biol Soc. 2006; 1:2694-7. 
10. Machado LS, Moraes RM, Nunes FLS. Serious Games para Saúde e Treinamento Imersivo. Sociedade Brasileira de Computação, 2009; 31-60.

11. Alves JC. Uso do Nintendo Wii como

Ferramenta de Treinamento da Funcionalidade, Equilíbrio e Qualidade de Vida em Idosos. Trabalho de Conclusão de Curso - Instituto Federal de Educação, Ciência e Tecnologia do Sul de Minas Gerais; 2013.

12. Young W, Ferguson S, Brault S, Craig C. Assessing and training standing balance in older adults: A novel approach using the 'Nintendo Wii' Balance Board. Gait\&Posture. 2O11; 33:303-305.
13. Kim J, Son J, Ko N, Yoon B. Unsupervised virtual reality-based exercise program improves hip muscle strength and balance control in older adults: a pilot study. ArchPhysMedRehabil. 2013; 94(5):937-943.

14. Treml CJ, Kalil Filho FA, Ciccarino RFL, Wegner RS, Saita CYS, Corrêa AG. O uso da plataforma Balance Board como recurso fisioterápico em idosos. Rev. Bras. Geriatr. Gerontol. 2013; 16(4):759-768.

15. Jones RJR, Dorgo S, Hitchings MK, Bader JO. WiiFitTM Plus balance test scores for the assessment of balance and mobility in older adults. Gait \& Posture. 2012; 36: 430-433. 